CM-P00062411

\title{
HIDDEN NON-COMPACT SYMMETRIES IN STRING THEORY ${ }^{*}$ )
}

\author{
S. Cecotti \\ CERN - Geneva \\ S. Ferrara and L. Girarde11o \\ CERN - Geneva \\ and \\ University of California, Los Angeles, CA 90024
}

and

\begin{abstract}
Four-dimensional (super) strings have an invariance at the classical level under a hidden non-compact symmetry. These symmetries are (explicitly) broken only by minimal gaugecouplings, as happens in supergravity. We discuss the dynamical origin of these symmetries at the level of the $2 d \sigma$-model describing the motion of a given string in an arbitrary background for the light modes. The hidden symmetries are a space-time manifestation of the non-compact, generalized duality rotations for the underlying interacting $2 d$ field theory.
\end{abstract}

*) This work is supported in part by the United States Department of Energy under Contract No. DE-AA03-76SF00034.

**) Permanent address: Università di Milano and INFN, Sezione di Milano, Italy. 


\section{Introduction.}

It is well known that many extended $4 \mathrm{D}$ supergravity theories have a large non-compact symmetry [1-6]. For historical reasons they are known as "hidden" symmetries [3]. Even if they are usually broken by the gauge-interactions, they are quite interesting since they determine the structure of the kinetic terms for all fields. In particular, in all known $N \geqslant 3$ supergravities the scalar's $\sigma$-model is given by a coset $G / H$ where $G$ is the non-compact hidden symmetry group and $H$ its maximal compact subgroup [4]. The specific cosets are listed in Table I.

For a supersymmetric 4D heterotic string [7], its effective low-energy theory $[8,9]$ is just a supergravity of the usual kind, and hence has such a hidden symmetry (which is explicitly broken by $O(g)$ terms). This symmetry again uniquely fixes the kinetic terms, and in particular, the full non-linear structure of the scalar's $\sigma$-model [8]. In Ref. 8 the relevant low-energy limits for $4 \mathrm{D}$ heterotic strings with $N \geqslant 1$ supersymmetry have been obtained using supergravity arguments and suitable $z_{2}$ projections of the massless spectrum (GSO projection [10]). The scalar fields (of the untwisted sector) parametrize manifolds of the type $S O(p, q) /[S O(p) \otimes S O(q)]$, where $p$ depends on the number of supersymmetries and $q$ on the gauge group.

A natural question is whether this (partial) hidden symmetry corresponds to an exact property of the string, ${ }^{*}$ or if it is just an artifact of the low-energy approximation. Indeed, some time ago Duff [11] conjectured that the (super-) strings do have non-compact hidden symmetries and also presented some preliminary evidence in their support.

\footnotetext{
*More precisely, an exact symmetry of a sector of the string.
} 
It is the purpose of the present article, to give a complete discussion of the dynamical origin of these partial symmetries in terms of the underlying $2 d$ $\sigma$-model describing the motion of the string in a generic background for the massless modes.

The knowledge of the microscopic origin of these symmetries greatly simplifies the calculation, from first string principles, of the full nonlinear effective Lagrangians. We shall discuss our general method for doing this elsewhere. The present results, even if they are mere algebraic lemmas of the general approach, suffice to compute the full structure of the kinetic terms in virtually all models (whether super-symmetric or not).

Interestingly enough it turns out that their two-dimensional origin is quite similar to the way hidden symmetries usually appear in 4D SUGRA, i.e. through generalized duality transformations $[2,3]$. The only difference is that in $2 d$ the vector field strength and its dual (which are two-forms) are replaced by a scalar field strength and its dual (one-forms). The crucial observation is now that, because commuting objects (2-forms) are replaced by anticommuting ones (1-forms) the maximal duality group is $S O(n, n)$ rather than $\operatorname{Sp}(2 n, R)$ as it is the case for $D=4[12]$.

The physical situation we want to study is the following: We have a fourdimensional ${ }^{*}$ string theory [7] moving in an arbitrary background for the light fields. The background is not required to be a solution of the relevant equation of motion. Then, the Lagrangian of the corresponding $\sigma$-model has the following general structure:

$$
\mathscr{L}=\mathscr{L}_{\mathrm{SP}}\left(\mathrm{X}^{\mu}\right)+\mathscr{L}_{\mathrm{INT}}\left(\mathrm{X}^{\mu}, \phi^{\mathrm{a}}\right)
$$

\footnotetext{
* Or any other dimension less than the critical one.
} 
where $\mathscr{L}_{\mathrm{SP}}\left(\mathrm{X}^{\mu}\right)$ is the Lagrangian describing the mechanical motion of the string in a four-manifold parametrized by the coordinates $x^{\mu}$, and $\mathcal{L}_{\operatorname{INT}}\left(\mathrm{X}^{\mu}, \phi^{2}\right)$ is the Lagrangian for the "internal" degrees of freedom $\phi^{a}$. The important point is that in a $4 \mathrm{D}$ string the background fields are functions of the coordinates $\mathrm{x}^{\mu}$ only, not of the "internal coordinates" $\phi^{a}$. Thus, for a given model, $\mathscr{L}_{\text {INT }}\left(\mathrm{X}^{\mu}, \phi^{\mathrm{a}}\right)$ has a fixed dependence on the internal fields $\phi^{\mathrm{a}}$, but an arbitrary one (through the background fields) on the $x^{\mu}$ 2d fields. For our purposes, we are interested only in a non-trivial background for scalars. All other fields can be put to zero (of course, our results are valid in the general case too). Then the background scalars may be seen--from the $2 d$ point of view--as $x^{\mu}$ dependent couplings of the $\phi^{\mathrm{a}}$ fields.

A symmetry of the effective Lagrangian has the following interpretation at the world-sheet level: there is a group of nonlinear transformations which map the given background into a new one, in such a way that the corresponding omodels are equivalent as $2 \mathrm{~d}$ field theories. In other words, there is a transformation of the $\mathrm{X}$-dependent couplings which leave the $2 \mathrm{~d}$ physics unchanged, irrespective of the particular dependence on the coordinates $x^{\mu}$ of the background fields.

Thus, showing the existence of hidden symmetries for the string amounts to proving some equivalence theorem in 2d QFT. We shall do this below by exploiting the generalized duality transformations. They were studied in the 4D case by Gaillard and Zumino [12] (see also Cremmer and Julia [2]). We start by extending their work to the $2 \mathrm{~d}$ case. Then we shall discuss the implications for the string case. 


\section{Generalized Duality Rotations in 2d.}

For simplicity, we start by considering only ordinary scalars; the case of chiral scalars, as well as the supersymmetric case, will be discussed later.

In $2 d$ the field strength of a scalar field $F_{\mu}{ }^{a}=\partial_{\mu} \phi^{a}$ satisfies the Bianchi identity

$$
\begin{aligned}
& \partial_{\mu} \tilde{F}^{\mathrm{a} \mu}=0 \\
& \tilde{\mathrm{F}}^{\mathrm{a} \mu}=\epsilon^{\mu \nu_{\partial} \phi^{\mathrm{a}} .}
\end{aligned}
$$

If the Lagrangian $\mathscr{L}$ depends on $\phi^{\mathrm{a}}$ only through their field strengths $\mathrm{F}_{\mu}{ }^{\mathrm{a}}$, the equations of motion read

$$
\begin{aligned}
& \partial_{\mu} \tilde{G}_{a}^{\mu}=0 \\
& \tilde{G}_{a}^{\mu}=\frac{\partial \mathscr{L}}{\partial F_{\mu}{ }^{a}} .
\end{aligned}
$$

Obviousoy, Eqs. (2) and (4) are invariant under any linear transformation among the F's and G's. However, as in Ref. 12, we are only interested in those transformations which are compatible with Eq. (5), and possibly with the equations of motion of other fields, and--most importantly--which are invariances of the energy-momentum tensor (i.e. of the conformal algebra).

In general, this will require that also other fields $\chi^{i}$ (typically scalars) transform under those duality transformations: $\delta \chi^{i}=\xi^{i}(\chi)$.

To find the relevant transformations, we have just to repeat, word by word, the analysis in Paragraph 2.1 of Ref. 12. There is a major difference however: in $4 \mathrm{D}$ we have $\mathrm{F}^{1} \tilde{F}^{2}=\mathrm{F}^{2} \tilde{F}^{1}$, whereas in $2 \mathrm{~d} \mathrm{~F}^{1} \tilde{F}^{2}=-\mathrm{F}^{2} \tilde{F}^{1}$. This 
introduces some extra minus sign in the formulas.

The generator $\mathrm{X}$ of the allowed transformations

$$
\begin{aligned}
& \delta_{X}\left(\begin{array}{l}
F \\
G
\end{array}\right)=x\left(\begin{array}{l}
F \\
G
\end{array}\right) \\
& \delta_{X} \chi^{i}=\xi_{X}^{i}(\chi)
\end{aligned}
$$

will satisfy the following conditions

$$
\mathrm{X}^{\mathrm{T}} \Omega=-\Omega \mathrm{X}
$$

where, in $4 \mathrm{D}$

$$
\Omega=\left(\begin{array}{rr}
0 & -1 \\
1 & 0
\end{array}\right)
$$

so that the duality group, for $n$ vectors, is (at most) $S p(2 n, R)$, whereas in $2 d$ case we have

$$
\Omega=\left(\begin{array}{ll}
0 & 1 \\
1 & 0
\end{array}\right)
$$

and the resulting duality group is $\operatorname{so}(n, n)$, or a subgroup.

The variation under (6) of the Lagrangian is

$$
\delta \mathscr{L}=\frac{1}{2}(\mathrm{FC} \tilde{F}+\tilde{G B G})
$$

where 


$$
X=\left(\begin{array}{cc}
A & B \\
C & -A^{T}
\end{array}\right)
$$

Using Eq. (10), it is easy to prove that the energy-momentum tensor is invariant under the transformation in Eq. (6).

The general form of the Lagrangian leading to invariant equations of motion is [12]

$$
\mathscr{L}=\frac{1}{2} \tilde{F G}+\mathscr{L}_{\text {inv }} .
$$

If the vectors $\left(\mathrm{H}_{\mu}{ }^{\mathrm{a}}(\chi), \mathrm{K}_{\mu \mathrm{a}}(\chi)\right)$ transform under (6) like $\left(\mathrm{F}_{\mu}{ }^{\mathrm{a}}, \mathrm{G}_{\mu \mathrm{a}}\right)$ we can have an interaction

$$
\mathscr{L}=\frac{1}{2} \tilde{F G}+\frac{1}{2}(\tilde{K F}+\tilde{H G})+\mathscr{L}_{i n v}(x)
$$

From Eqs. (5) and (13) we get an equation for G

$$
*\left(G_{a}+K_{a}\right)=\left[g_{a b}(\chi)+b_{a b}(\chi)^{*}\right]\left(F^{b}+H^{b}\right)
$$

for some matrices $g$ (positive) and $b\left(g^{T}=g, b^{T}=-b\right)$.

From Eqs. (12) and (13) we have

$$
\begin{aligned}
& \mathscr{L}=\frac{1}{2} g_{a b} \partial^{\mu} \phi^{a} \partial_{\mu} \phi^{b}+\frac{1}{2} b_{a b} \varepsilon^{\mu \nu} \partial_{\mu} \phi^{a} \partial_{\nu} \phi^{b}+g_{a b} \partial^{\mu} \phi^{a_{H}}{ }_{\mu}^{b}+b_{a b} \varepsilon^{\mu \nu} \partial_{\mu} \phi^{a_{H}}{ }_{\nu}^{b} \\
& -\varepsilon^{\mu v} \partial_{\mu} \phi^{a} K_{a v}+\frac{1}{2} g_{a b} H^{\mu a_{H}}{ }_{\mu}^{b}+\frac{1}{2} b_{a b} \varepsilon^{\mu v_{H}}{ }_{\mu}^{a_{H}}{ }_{v}^{b} \\
& -\frac{1}{2} \varepsilon^{\mu v_{H}}{ }_{\mu}^{a} K_{v a}+\mathscr{L}_{i n v}(\chi)
\end{aligned}
$$


As in 4D [2], Eq. (14) can be rewritten in an $S O(n, n)$ covariant way. Let

$$
\hat{f}=\left(\begin{array}{l}
F^{a}+H^{a} \\
G_{a}+K_{a}
\end{array}\right)
$$

then Eq. (14) is equivalent to [2]

$$
\hat{V F}=\Omega \tilde{\hat{F}}
$$

where the matrix $v=r(x)$ is an $\operatorname{SO}(n, n)$ group element

$$
t_{r \Omega v}=\Omega
$$

Eq. (17) is invariant under

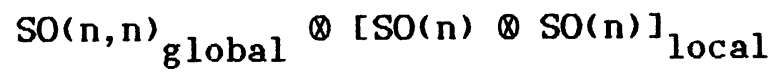

where $\operatorname{SO}(n, n)$ acts as

$$
V \rightarrow V_{g}^{-1}, \quad F \rightarrow g F
$$

and $[S O(n) \times S O(n)]_{10 c a l}$ as

$$
r \rightarrow \mathrm{h} v,
$$

here $[S O(n) \times S O(n)]]_{\text {local }}$ is the maximal compact subgroup of $\operatorname{SO}(n, n)$, corresponding to the elements $h$ commuting with $\Omega$.

Comparing Eqs. $(17, \ldots, 21)$, with Eq. (14) we see that the matrix $\mathrm{T}_{\mathrm{ab}}=$ $g_{a b}(\chi)+b_{a b}(\chi)$ parametrizes the $\operatorname{coset} S O(n, n) /[S O(n) \otimes S O(n)]$. The 
precise correspondence is

$$
T=\left(v_{4}-v_{2}\right)^{-1}\left(v_{1}-v_{3}\right)
$$

where

$$
v=\left(\begin{array}{ll}
v_{1} & v_{2} \\
v_{3} & v_{4}
\end{array}\right)
$$

The (nonlinear) action of $S O(n, n)$ on $T$ is obvious from Eqs. (20) and (22). Next, we consider the case of chiral bosons [13].

Suppose we have $p$ left moving $\left(\phi^{a}\right)$ and four right-moving $\left(\phi^{i}\right)$ scalars. Their Lagrangian is similar to Eq. (14), but with some Siegel terms [13] enforcing the (anti-) self duality constraint. In all the relevant models, these constraints can be put into the form

$$
\begin{array}{ll}
F_{+}{ }^{i}+G_{+i}=0, & i=1, \ldots, q \\
F_{-}{ }^{a}-G_{-a}=0, & a=1, \ldots, p .
\end{array}
$$

In order not to have spurious propagation, the corresponding Lagrange multipliers should be just gauge degrees of freedom, that can be gauged away by a Siegel-like [13] transformation. [Of course, these requirement of gaugeinvariance give severe constraints on the possible interactions.]

Apart from Eq. (24), all the other equations of motion can be put in the same form as before. Consequently, the duality group in the chiral case is just that subgroup of the general duality $\mathrm{SO}(\mathrm{p}+\mathrm{q}, \mathrm{p}+\mathrm{q})$ group of Eqs. (19 and (20) which preserves Eqs. (24). By going to a basis in which $\Omega$ is diagonal, 
this group is easily seen to be

$$
G=S O(q) \otimes S O(p, q) \otimes S O(p)
$$

The two compact factors in Eq. (25) are physically irrelevant and can be ignored. Thus we have the symmetry (20) with $g \in G$ and also the local symmetry (21) with $h \in S O(q)^{2} \otimes S O(p)^{2}$. Then the self-couplings (compatible with duality) of the chiral bosons are naturally described in terms of the coset

$$
\mathrm{G} / \mathrm{H}=\mathrm{SO}(\mathrm{p}, \mathrm{q}) / \mathrm{SO}(\mathrm{p}) \otimes \mathrm{SO}(\mathrm{q})
$$

The same result can also be obtained by an explicit construction of the Siegelinvariant Lagrangians corresponding to the coupling of left and right moving scalars.

The above analysis can be repeated, almost word by word in the SUSY case. But now, besides the energy-momentum tensor, also the supercurrent(s) are duality-invariant.

Here we limit ourselves to the case of $(1,0)$ SUSY, which is the one relevant for the heterotic string. Both the $\phi^{\prime} s$ and the $\chi$ 's get promoted to $(1,0)$ superfields [14]. We define the superspace field-strengths $F_{A}{ }^{a}$ as

$$
\begin{aligned}
& F_{+}{ }^{a}=i D_{+} \phi^{a}, \quad F_{--}{ }^{a}=\partial_{--} \phi^{a} \\
& F_{++}{ }^{a}=D_{+}\left(F_{+}{ }^{a}\right) .
\end{aligned}
$$

The superspace Lagrangian $\mathscr{L}_{S}$ (which is a spinor) is 


$$
\mathscr{L}_{s}=\mathscr{L}_{s}\left(F_{+}^{\mathrm{a}}, \mathrm{F}_{--}^{\mathrm{a}}, x\right)
$$

Defining

$$
\frac{\partial \mathscr{L}_{\mathbf{s}}}{\partial F_{+}^{\mathrm{a}}}=-\mathrm{G}_{--} \mathrm{a}, \frac{\partial \mathscr{L}_{\mathbf{s}}}{\partial \mathrm{F}_{--} \mathrm{a}}=\mathrm{G}_{+}^{\mathrm{a}}
$$

we have the equations of motion

$$
\partial_{--} G_{+}^{a}-i D_{+} G_{--}{ }^{a}=0
$$

and the Bianchi-identities

$$
\partial_{--} F_{+}^{a}-i D_{+} F_{--}^{a}=0
$$

Defining also

$$
G_{++}^{a} \equiv D_{+}\left(G_{+}^{a}\right)
$$

we have that all the equations, $(28,31)$ are invariant under any linear transformations among the superfield strengths $F_{A}{ }^{a}$ and $G_{A}{ }^{a}$. But, again, only a restricted set are meaningful in the interacting theory and invariances of the supercurrent.

Defining

$$
\begin{aligned}
& \tilde{F G} \equiv G_{+} F_{--}-F_{+} G_{--} \\
& \tilde{F G}=-\tilde{G F} .
\end{aligned}
$$


We see that all the formal manipulations we need are the same as in the bosonic case, and thus we obtain the same result, Eqs. (6), (7), and (9). The same conclusion holds for the supersymmetric theory of chiral bosons. As above we obtain a duality symmetry $G_{g l o b a l} \otimes H_{10 c a l}$, with $G=S O(p, q)$ and $H=S O(p) \times S O(q)$. Thus the quadratic couplings are parametrized by superfields taking values in the coset $G / H$, as before.

Do the above non-compact symmetries survive the quantization of the $2 d \sigma-$ model? For the applications to the string we need a weaker notion of invariance, namely that the effective action

$$
\begin{aligned}
& \exp \left\{\mathrm{iWC} \mathrm{g}_{\mathrm{ab}}, \mathrm{b}_{\mathrm{ab}}, \mathrm{H}_{\mu}{ }^{\mathrm{a}}, \mathrm{K}_{\mathrm{a} \mu}{ }^{]\}}\right. \\
& =\int[\sqrt{\operatorname{detg}} \mathrm{d} \phi] \exp \left\{\mathrm{i} \int\left[\frac{1}{2} \tilde{\mathrm{FG}}+\frac{1}{2}(\tilde{\mathrm{KF}}+\tilde{H G})\right] \mathrm{d}^{2} \mathrm{z}\right\} .
\end{aligned}
$$

is invariant when the couplings $\mathrm{g}_{\mathrm{ab}}, \mathrm{b}_{\mathrm{ab}}, \mathrm{H}_{\mu}^{\mathrm{a}}$ and $\mathrm{K}_{\mu \mathrm{a}}$ (considered as background fields with a given dependence on the $2 d$ coordinates) are transformed under $G_{\text {global }}$ as in Eqs. (20), (22) and (23). If the world-sheet has $H^{1}=0$ (i.e. on the sphere) this can be easily seen by direct path-integral manipulations. They are essentially the same formal tricks as employed by Fradkin and Tseytlin [15] to prove the quantum equivalence of many dual field theories. The manipulations are simplified by the use of the manifestly SO $(p, q)$ invariant action principle of Cremmer and Julia [6]. Thus

$$
\begin{aligned}
& W=W\left[r d r^{-1}, r x_{\mu}\right] \\
& x_{\mu} \equiv\left(\begin{array}{l}
H_{\mu}^{a} \\
K_{\mu}^{a}
\end{array}\right) .
\end{aligned}
$$


Eq. (33) may be seen as an equivalence theorem for the models in Eq. (15), whose background fields $\mathrm{g}, \mathrm{b}, \mathrm{H}$, and $\mathrm{K}$ belong to the same $\mathrm{SO}(\mathrm{p}, \mathrm{q})$ orbit.

On the contrary, for Riemann surfaces with $g \geqslant 1$, $S O(p, q)$ is not a symmetry in the sense of Eqs. (33), as can be easily argued from Eq. (10). Then, in the string langauge, $\mathrm{SO}(\mathrm{p}, \mathrm{q})$ is a classical symmetry.*

\section{Applications to (Super-) Strings.}

We are ready to discuss the implications of $2 d$ duality transformations for the classical effective Lagrangian of a given string theory. We begin with the simplest case, i.e. a 4D (heterotic) string with $N=4$ supersymmetry. To fix the ideas, let us consider the model with gauge group SO(44) (or $\mathrm{E}_{6} \otimes \mathrm{E}_{8} \otimes$ $E_{8}$ ) [7]. The effective Lagrangian for these models was computed, using indirect methods, in Ref. 8.

Of course, this model has no exact non-compact symmetry. Indeed, from simple supergravity arguments [16], we know that the hidden symmetry must be explicitly broken by terms in the effective Lagrangian proportional to the gauge coupling $\mathrm{g}$. Then, it is convenient to start with the simplified situation in which the hidden symmetry is an exact invariance of the effective Lagrangian, higher-derivative terms included. This can be obtained in the following way: all the (space-time) matter fields belong to the adjoint representation of the gauge group. If we limit ourselves to the class of backgrounds in which** only the fields corresponding to the Cartan subalgebra have non-trivial values, then the truncated effective Lagrangian is truly invariant under the non-compact symmetry to all orders (both in fields and

\footnotetext{
*In general, the local symmetry $S O(p) \times S O(q)$ may be anomalous, but, in the string applications, this anomaly is likely to be cancelled by the $\sigma$-model version of the Green-Schwarz mechanism [16].

**Apart from the gravitational fields $\left(g_{\mu v}, b_{\mu v}, \phi\right)$ which are still arbitrary.
} 
their derivatives). Why it is so, it is obvious from a $\sigma$-model point of view. Consider the 2d Lagrangian for the So(44) model, moving in an arbitrary background, as given by Gates and Siegel [17]. In this formulation, $\mathscr{L}_{\text {INT }}(\mathrm{x}, \phi)$ is described as a system of six $(1,0)$ leftons coupled through the background fields (gauge-scalars) to 44 right-moving fermions. If the background fields are restricted to be "Abelian", we can get rid of the 44 fermions by Abelian bosonization [18]. Then we replace the 44 Majorana-Weyl fermions with 22 $(1,0)$ rightons moving on a flat torus. The Lagrangian then reads (in the $(1,0)$ superspace $[14])$

$$
\begin{aligned}
\mathscr{L}_{\text {INT }} & =\int d \theta L_{\text {INT }}, \\
L_{\text {INT }} & =1 D_{+} Y^{a}\left[\partial_{--} Y^{a}+2 A_{a i}(X) \partial_{--} \phi^{i}\right]+i D_{+} \phi^{i} \partial_{--} \phi^{i} \\
& + \text { (Fadeev-Popov ghosts for the Siegel-like gauge symmetry) }
\end{aligned}
$$

where $\mathrm{Y}^{\mathrm{a}}, \mathrm{a}=1,-, 6$ are the leftons corresponding to the "internal" coordinates and $\phi^{i}, i=1, \ldots, 22$ are the rightons bosonizing the 44 right-moving fermions. The coupling $A_{a i}\left(x^{\mu}\right)$ are the background scalars, belonging to the SO(2) $)^{22}$ subgroup of SO(44). Here $X^{\mu}$ is also a $(1,0)$ superfield.

One can show that Eq. (34) is invariant under the BRS transformations corresponding to the Siegel gauge-invariance [13]. Moreover, the coupling to 2d supergravity causes no fundamental problem, if the $\sigma$-model corresponds to a modular invariant string theory.

The classical equations of motion are

$$
\partial_{--} Y^{a}+A_{a i}(X) \quad \partial_{--} \phi^{i}=0
$$




$$
D_{+} \phi^{i}+A_{a i}(X) D_{+} Y^{a}=0
$$

which are (of course) of the general form in Eq. (24)--except that now we work in the $(1,0)$ superspace.

From our discussion of generalized duality, we see that if in the $\sigma$-model (34), we change the background fields $A_{a 1}(x)$ by a (nonlinear) $\operatorname{SO}(6,22)$ transformation, we get a $\sigma$-model which--as a $2 d$ field theory--is equivalent to the original one. To be more concrete, let $v(x)$ be the element of the SO $(6,22)$ group (on the basis that $\Omega$ is diagonal) and let

$$
F_{A}=\left(\begin{array}{l}
F_{A}^{a}+G_{a A} \\
F_{A}^{i}-G_{i A}
\end{array}\right),
$$

$(A=+,--)$ be the combinations of the F's and G's which are orthogonal to the chirality constraints (Eqs. (24) and (35)), i.e. the combinations which do not vanish on-shell. Then Eq. (17), which is the relation which defines the G's as functions of the F's and the background, becomes

$$
r(X) F_{A}=\varepsilon_{A} \Omega r(X) F_{A}
$$

where $\varepsilon_{A}=+1$ for $A=+$ and -1 for $A=--$.

Comparing Eq. (37) with Eqs. (34), (35) and (24), we get the following expression for the background matrix $A(x)$ in terms of the group element

$$
A(X)=\left[v_{1}(X)\right]^{-1} v_{2}(X)=\left\{\left[v_{4}(X)\right]^{-1} v_{3}(X)\right\}^{T}
$$

where the $v_{i}$ 's are defined as in Eq. (23), but now on a basis on which $\Omega$ is diagonal. 
Again, Eq. (37) has a global SO(6,22) symmetry acting as

$$
r(X) \rightarrow r(X) g^{-1}, \quad F_{A} \rightarrow g^{\mathcal{F}} A
$$

and a local $\mathrm{SO}(6) \otimes \mathrm{SO}(22)$ symmetry

$$
r(X) \rightarrow h(z, \bar{z}) r(X),
$$

which leaves $A(x)$, Eq. (38), invariant. Thus the background $A(x)$ lives on the coset $S O(6,22) / S O(6) \otimes S O(22)$. Moreover, two backgrounds $A(x)$ and $A^{\prime}(x)$ which differ by a SO $(6,22)$ transformation as in Eqs. (38) and (39) correspond to equivalent $\sigma$-models. Then the effective Lagrangian (truncated to the Abelian sector) is invariant under the hidden $S O(6,22)$ group, which acts on the scalars $A_{a i}(X)$ as

$$
A^{\prime}(X)=\left[\alpha^{T}-A(X) \beta^{T}\right]^{-1}\left[A(X) \delta^{T}-\gamma^{T}\right]
$$

if

$$
g=\left(\begin{array}{ll}
\alpha & \beta \\
\gamma & \delta
\end{array}\right)
$$

In particular, the above equivalence theorem implies that the kinetic term for the scalars $A_{\alpha i}(X)$ corresponds to the $\sigma$-model with the unique $\operatorname{SO}(6,22)-$ invariant metric on the coset $\mathrm{SO}(6,22) / \mathrm{SO}(6) \times \mathrm{SO}(22)$. But, in fact, we have proved much more, since this is an exact symmetry of the (classical) effective theory, in the Abelian sector. In particular it is a symmetry of the higher derivative terms [19]. 
The same conclusion holds if we allow a non-trivial background for the graviphotons and the gauge vectors in the Cartan subalgebra. The only change is that we have a non-vanishing $\mu_{A}$ (see Eqs. (33c), (13)-(16)).

At this point it is worth noticing that the same coset and (fourdimensional) $\sigma$-model, is obtained if one compactifies the heterotic string on a flat torus (and truncate to the Abelian configurations). In this case the background fields are*

$$
\begin{aligned}
& T_{a b}(X)=g_{a b}(X)+b_{a b}(X), \quad a, b=1, \ldots, 6 \\
& A_{a i}(X) \quad, \quad i=7, \ldots, 22
\end{aligned}
$$

and we have 6 ordinary bosons (the internal coordinates) and 16 rightons. It appears that 6 leftons and 6 rightons have been combined into 6 ordinary boson superfields. Indeed, the two interacting $2 d$ theories can be shown to be equivalent (by the same methods as in the discussion after Eq. (34)), if $\mathrm{T}$ is related to $A_{a i}, i=1, \ldots, 6$ as

$$
T_{a b}(X)=[1-A(X)][1+A(X)]_{a b}^{-1}
$$

and a corresponding transformation for the $A_{a i}, i=7, \ldots, 22$. These transformations were noticed before (see Ref. 20) and used to put the Lagrangian for the string compactified on a torus in a manifestly invariant SO(6, dimG) form [20]. Here we understand this nonlinear transformation of variables $\mathrm{T} \rightarrow \mathrm{A}$, which puts the low energy kinetic terms in a canonical form,

\footnotetext{
*Same notation as in Eqs. (14), (15) and (22).
} 
as a consequence of an exact equivalence theorem between a 2d theory with 6 ordinary bosons and a theory with 6 leftons and 6 rightons. Details and further applications will be provided elsewhere.

In the particular case in which the fields (43) are just constants, we get a free theory describing new string vacua. Then, it is obvious that these vacua are parametrized by the coset $\mathrm{SO}(6,22) / \mathrm{SO}(6) \otimes \mathrm{SO}(22)$ with the symmetry groups acting on the background fields as follows from Eqs. (38) and (43). Indeed, this is exactly the Narain construction of new string theories [21]. The full Narain spectrum (including the additional gauge bosons) can be recovered by studying the quantum duality transformation on a world-sheet with the torus topology (this is quite easy to do in the free case). We think that our procedure has the merit of making the action of the non-compact gauge group on the background fields explicit and also of clarifying how this group can be seen as rotations between left- and right-moving bosons.

Now we turn to the general case, i.e. all fields in the background have non-trivial values. The main difference is that now we need the non-Abelian bosonization [22], instead of the Abelian one. Eq. (34) gets replaced by (see also Ref. 17)

$$
\begin{aligned}
L_{I N T}= & -\frac{i}{2 \pi} \operatorname{Tr}\left\{J_{--}\left[J_{+}+2 \hat{A}^{a}(X) D_{+} Y^{a}\right]\right\} \\
& +\frac{i}{2 \pi} \int_{0}^{1} d t \operatorname{Tr}\left\{\left(R^{-1} \partial_{t} R\right)\left[\partial_{--} J_{+}-D_{+} J_{--}\right]\right\}+\frac{1}{2 \pi} D_{+} Y^{a_{2} \partial_{--} Y^{a}} \\
& + \text { (ghost and gauge-fixing terms) . }
\end{aligned}
$$

Here $R$ is a right-moving field taking value on the gauge group manifold (SO (44) or whatever else) and the current $J_{A}$ is defined as $R^{-1} D_{A} R$. The 
coupling $\hat{A}^{a}(X)=R^{-1} A^{a}(X) R$, with $A^{a}(X)$ taking value in the gauge group Lie algebra

$$
A^{a}(X)=i T_{i} A^{a i}(X)
$$

$A^{a i}(X)$ is the scalars' background. Notice that an arbitrary background is compatible with Siegel gauge-invariance [13] only for a Kac-Moody of level one.

Now, the equations of motion for this model take, in terms of the nonAbelian currents $\mathrm{J}_{\mathrm{A}}$ and their variational duals, the same form as in the case (34) in terms of the Abelian currents $D_{A} \phi^{i}$, Eq. (35). However, in the non-Abelian case, the Bianchi identities get replaced by the Maurer-Cartan identities, which contain also a term proportional to the commutator of currents. This commutator term spoils the invariance under rotations of the currents into their "duals." However, if we rescale the generators, this commutator has formally a gauge coupling constant $g$ in front of it. Then, if we are allowed to take the limit $g \rightarrow 0$ while keeping the Planck mass fixed (since it is just the parameter which controls the nonlinearity in the kinetic terms), we would conclude that in this limit (which does not affect the kinetic terms), we obtain the same result as in the Abelian case, but this time with dim $G_{\text {gauge }}$ rightons. Indeed, in this limit the group manifold gets contracted into a flat space, in which our previous arguments apply. One can wonder if such a limit exists, since by the quantization of the coefficient in the Wess-Zumino Lagrangian [22], $g$ and the Planck mass are not independent. However, there is a "free" parameter, namely the v.e.v. of the dilaton. By rescaling both the gauge generators and the dilaton expectation value, we can make the (effective) gauge coupling constant small [23], while keeping the 
Planck mass (and the vector kinetic terms) fixed.

As a conclusion, in the general case, the kinetic term sector retains the non-compact symmetry, which is violated (in the effective Lagrangian) only by terms $0\left(g^{e f f}\right)$.

Then, the light scalar fields of any $\mathbf{N}=4$ heterotic string parametrize the coset

$$
\mathrm{SO}(6, \operatorname{dim} \mathrm{G}) / \mathrm{SO}(6) \otimes \mathrm{SO}(\operatorname{dim} G)
$$

and their kinetic terms are based on the invariant metric on this manifold. The same conclusion was obtained in Ref. 8 using macroscopic techniques [5]. Moreover, the vectors' kinetic terms also agree with Ref. 8.

The same strategy as above can be also used for $N<4$ superstring, and for non-supersymmetric strings as well (for instance, the compactified bosonic string ).

We are not giving here a full zoology of the effective Lagrangians here for the 4D strings [7]. However, we shall give a few hints of how, without much effort, one can construct the kinetic term sector of virtually all models by simple use of generalized duality invariance and equivalence rules. More powerful methods, allowing also the computation of the $O(g)$ terms, will be discussed elsewhere.

Many 4D strings [7] differ from the above example just because of a different (generalized) GOS projection [10]. The corresponding $2 \mathrm{~d} \sigma-$ model is then similar to the previous one, the only difference being that the only background field which are allowed to be non-zero are those which couple to the 
2d o-model through an admissible operator* (0-picture [24] vertex).

Thus, in many situations, the $\sigma$-models for the given $N<4$ (heterotic) strings can be seen as just the $\sigma$-model for the So(44) string in a special class of backgrounds, namely those in which the "non-admissible" fields have vanishing values. For a general (heterotic) string, this is the $\sigma$-model for at least a sector of the theory (it is the analog of the Abelian truncation we used before).

These restrictions on the background lead to a further condition on the group element $r(X)$, which now should belong to the subgroup of SO(6, dim SO(44)) commuting with the GOS projector $P$. Then, the net result is that the hidden symmetry of (a sector of) the theory is the proper subgroup G' $\subseteq$ SO(6, dim SO(44)), such that G'P = PG'. The 4d $\sigma$-model for the strings with $N$ $<4$ is based on the coset $G^{\prime} / H^{\prime}$, where $H^{\prime}$ is the maximal compact subgroup of G'. It is a general consequence of the theorem [25] relating the number of space-time symmetries $N$, with the number of world-sheet ones, that in the sector so obtained G' has the general form:

$$
\mathrm{G}^{\prime}=\mathrm{SO}(2, \mathrm{n}) \otimes \mathrm{SO}(4, \mathrm{~m})
$$

for an $N=2$ (heterotic) string;

$$
\mathrm{G}^{\prime}=\mathrm{SO}(2, \mathrm{n}) \otimes \mathrm{SO}(2, \mathrm{~m}) \otimes \mathrm{SO}(2, \ell)
$$

for an $N=1$ (heterotic) string;

\footnotetext{
*A $2 d$ operator is called admissible if it commutes with the GOS projection for the given string model.
} 
[Moreover, in all these examples, there is a further SU(1,1) hidden symmetry, connected to the coset $S U(1,1) / U(1)$ on which lives the dilaton. The $2 d$ origin of this non-compact symmetry is very easy to understand, but its discussion requires more general techniques and will be presented elsewhere.]

In this way we obtain full agreement with the results of Ref. 8 .

One can also compute quite easily, the effective Lagrangian for the heterotic string compactified on a torus and modded out by a $\mathrm{Z}(3)$ symmetry (see Ref. 20 for an indirect computation). In this case the constraint on the allowed backgrounds requires the internal coordinates to be complex [20]. The subgroup of $S O(p, q)$ commuting with the complex structure is obviously $\mathrm{SU}(\mathrm{p} / 2, q / 2)$ so that (for $p=6$ ) we get the coset

$S U(3, n) / S U(3) \otimes S U(n) \otimes U(1)$

in agreement with Ref. 20. Analogously, modding out SU(3), as in the original Witten [26] example, we get $S U(1, n) / S U(n) \otimes U(1)$. To obtain this result, one implicitly uses the equivalence theorem to relate $2 d \sigma$-models describing [27] a compactification à la Kaluza-Klein on a manifold of metric $g_{a b}$ and internal 2-form $b_{a b}$ with an equivalent system of coupled leftons-rightons, see Eq. (44).

There is another important implication of our analysis for the low-energy effective Lagrangian. For brevity, we discuss here only the simplest case, i.e. the Witten model [26]. In this case, the Kähler potential in a first approximation reads

$$
\tilde{K}=-3 \ln \left(T+\bar{T}-2 \bar{C}^{a} C_{a}\right)
$$


A priori this form can be modified by higher o-model loop terms. Using only ordinary (i.e. not hidden) symmetries, one restricts the general form of the correction to [28], [29] (neglecting string loop effects).

$$
\tilde{K}=-3 \ln \left(T+\bar{T}-2 \bar{C}^{a} C_{a}\right)+\bar{K}[C / \sqrt{T+\bar{T}} ; \bar{C} / \sqrt{T+\bar{T}}]
$$

for some function $\bar{K}$. Then the hidden symmetry implies: (1) if we restrict the chiral fields $T, C^{a}$ to belong to an "Abelian" configuration* $\bar{K} \equiv 0$; this puts severe restrictions on the group-invariants appearing in $\bar{K}$; and $(2) \bar{K}=O(g)$, i.e. $\overline{\mathrm{K}}$ vanishes in the formal limit $\mathrm{g} \rightarrow 0$.

We think that the above (simple) examples well illustrate the interest and the computational power of the non-compact hidden symmetries for understanding the classical nonlinear physics of a string theory.

\section{References.}

[1] E. Cremmer, J. Scherk, and S. Ferrara, Phys. Lett. 74B (1978) 61.

[2] E. Cremmer and B. Julia, Nucl. Phys. B159 (1979) 141.

[3] E. Cremmer, in Supergravity '81, ed. S. Ferrara and J. G. Taylor (Cambridge University Press, Cambridge, 1982), p. 313; E. Cremmer, in Supersymmetry. A Decade of Development, ed. P. C. West (Adam Hilger, Bristol and Boston, 1986), p. 289.

[4] J. P. Derendinger and S. Ferrara, in Supersymmetry and Supergravity '84, ed. B. de Wit, P. Fayet and P. van Nieuwenhuizen (World Scientific, Singapore, 1985), p. 159.

\footnotetext{
*An "Abelian" configuration is one obtained by SU(3) truncation of an Abelian one in the previous sense.
} 
[5] M. De Roo, Nucl. Phys. B255 (1985) 515; Phys. Lett. 156B (1985) 331;

E. Bergshoeff, I. G. Koh, and E. Sezgin, Phys. Lett.

M. De Roo and P. Wagemans, Nucl. Phys. B262 (1985) 646.

[6] L. Castellani, A. Ceresole, S. Ferrara, R. D'Auria, P. Fré, and E. Maina, Nucl. Phys. B268 (1986) 317; Phys. Lett. 161B (1985) 91.

[7] K. S. Narain, Phys. Lett. 169B (1986) 61; K. S. Narain, M. H. Sarmadi, and E. Witten, Nuc1. Phys. B279 (1987) 369; W. Lerche, D. Lust, and A. N. Schellekens, Nucl. Phys. B287 (1987) 477; M. Kawai, D. C. Lewellen and S. H. H. Tye, Phys. Rev. Lett 57 (1986) 1832; Nucl. Phys. B288 (1987) 1; M. Mueller and E. Witten, Phys. Lett. B182 (1986) 28; I. Antoniadis, C. Bachas, and C. Kounnas, Nucl. Phys. B289 (1987) 87; K. S. Narain, M. H. Sarmadi, and C. Vafa, Nucl. Phys. B288 (1987) 551; I. Antoniadis and C. Bachas, Preprint CERN-TH. 4767/87.

[8] S. Ferrara, L. Girardello, C. Kounnas, and M. Porrati, Phys. Lett. 192B (1987) 368; Phys. Lett. B194 (1987) 358; I. Antoniadis, J. Ellis, E. Floratos, D. V. Nanopoulos, and T. Tomaras, Phys. Lett. 191B (1987) 96.

[9] J. Lauer, D. Lüst, and S. Theisen, MPI-PAE/PTh 86/87.

[10] F. Gliozzi, J. Scherk, and D. Olive, Nucl. Phys. B122 (1977) 253;

N. Seiberg and E. Witten, Nucl. Phys. B276 (1986) 272.

[11] M. Duff, Phys. Lett. 173B (1986) 289.

[12] M. K. Gaillard and B. Zumino, Nucl. Phys. B193 (1981) 221.

[13] W. Siegel, Nucl. Phys. B238 (1984) 207; J. M. F. Labastida and M. Pernici, Nucl. Phys. B297 (1988) 557.

[14] R. Brooks, F. Muhammad, and S. Gates, Nuc1. Phys. B268 (1986) 599.

[15] E. S. Fradkin and A. A. Tseytlin, Ann. Phys. (N.Y.) 162 (1985) 31. 
[16] M. B. Green and J. H. Schwarz, Phys. Lett. 149B (1985) 117;

C. H. Hull and E. Witten, Phys. Lett. 160B (1985) 398; L. Bonora,

P. Cotta-Ramusino, M. Rinaldi, and J. Stasheff, Comm. Math. Phys. $112(1987) 237$ and to appear.

[17] S. J. Gates and W. Siegel, Preprint UMDEPP 88-113 (November 1987).

[18] S. Coleman, Phys. Rev. D11 (1975) 2088; S. Mandelstam, Phys. Rev. D11 (1975) 3026.

[19] S. Cecotti, S. Ferrara, L. Girardello, and M. Porrati, Phys. Lett. 185B (1987) 345.

[20] S. Ferrara, C. Kounnas, and M. Porrati, Phys. Lett. B181 (1986) 263.

[21] K. S. Narain, Phys. Lett. 169B (1986) 41; K. S. Narain, M. H. Sarmodi, and E. Witten, Nucl. Phys. B279 (1987) 369.

[22] E. Witten, Comm. Math. Phys. 92 (1984) 455; P. Di Vecchia, V. G. Knizhnik, J. L. Petersen, and P. Rossi, Nucl. Phys. B253 (1985) 701.

[23] M. Dine, R. Rohm, N. Seiberg, and E. Witten, Phys. Lett. B156 (1985) 457.

[24] D. Friedan, E. Martinec, and S. Shenker, Nucl. Phys. B271 (1986) 93.

[25] W. Boucher, D. Friedan, and A. Kent, Phys. Lett. B172 (1986) 316;

A. Sen, Nucl. Phys. B278 (1986) 289; B284 (1987) 423; L. Dixon,

D. Friedan, and S. H. Shenker, Nucl. Phys. B282 (1987) 13;

T. Banks, L. J. Dixon, D. Friedan, and E. Martinec, Preprint SLAC-PUB4377 (1987); S. Cecotti, S. Ferrara, L. Girardello, Preprint UCLA/88/ TEP/10; CERN-TH-4982.

[26] E. Witten, Phys. Lett. 155B (1985) 151.

[27] A. Sen, Nucl. Phys. B278 (1986) 289; B284 (1987) 423. 
[28] C. P. Burgess, A. Font, and F. Quevedo, Nucl. Phys. B272 (1986) 661; H. P. Nilles, Phys. Lett. 180B (1986) 240.

[29] S. Cecotti, S. Ferrara, and M. Villasante, Int. J. Mod. Phys. A2 (1987) 1839. 
TABLE I : THE SCALARS' $\sigma$-MODEL COSETS FOR EXTENDED SUGRA

$\underline{\mathbf{N}}$

$\underline{\text { Coset }}$

$3 \quad \mathrm{SU}(3, \mathrm{n}) /[\mathrm{SU}(3) \otimes S U(n)] \sim U(1)$

$4\{\operatorname{SO}(6, n) /[S O(6) \otimes S O(n)]\} \otimes\{S U(1,1) / U(1)\}$

$5 \quad \mathrm{SU}(5,1) /[\mathrm{SU}(5) \otimes U(1)]$

$6 \mathrm{SO}^{*}(12) /[\mathrm{SU}(6) \otimes \mathrm{U}(1)]$

$8 \quad E_{7,(+7)} / \mathrm{SU}(8)$ 